\title{
Fachspezifität von Unterrichtsqualität in der Deutschdidaktik
}

\author{
Maja Wiprächtiger-Geppert $\mathbb{D} \cdot$ Ruven Stahns $\cdot$ Susanne Riegler
}

Eingegangen: 14. Dezember 2020 / Überarbeitet: 19. April 2021 / Angenommen: 28. April 2021 / Online publiziert: 4. Juni 2021

(C) Der/die Autor(en) 2021

Zusammenfassung Ausgehend von der Beobachtung, dass sich die Deutschdidaktik in der Vergangenheit nur implizit mit Unterrichtsqualität auseinandergesetzt hat, werden in diesem Beitrag aktuelle Tendenzen der deutschdidaktischen Forschung in Bezug auf Unterrichtsqualität dargestellt. Dabei kann man feststellen, dass es mittlerweile einige Studien gibt, die an Befunde der allgemeinen Unterrichtsqualitätsforschung anschließen und diese vor allem mit Blick auf das Merkmal ,kognitive Aktivierung “ für den Deutschunterricht spezifizieren. Dabei handelt es sich zum einen um Fragebogenstudien im Rahmen von Large-Scale-Assessements, die eher allgemein gehaltene Merkmale von Unterrichtsqualität auf den Deutschunterricht beziehen und damit fachbezogen sind. Zum anderen handelt es sich um Videostudien, in denen kognitive Aktivierung nicht mehr allgemein, sondern fachspezifisch konzipiert und operationalisiert wird. Im zweiten Teil wird das im Projekt „Profess-R“ verwendete Konzept fachspezifischer Unterrichtsqualität kurz präsentiert. Ausgehend von der Überzeugung, dass für eine angemessene fachspezifische Konturierung von Unterrichtsqualität die Spezifizierung von kognitiver Aktivierung nicht ausreichend ist, wird ein Modell vorgestellt, in dem generische, fachbezogene und fachspezifische Aspekte auf mehreren Ebenen ineinandergreifen.

Schlüsselwörter Fachspezifische Unterrichtsqualität · Deutschdidaktik · Kognitive Aktivierung $\cdot$ Rechtschreibunterricht

Die Autor(inn)en haben zu gleichen Teilen zu diesem Text beigetragen.

Maja Wiprächtiger-Geppert $(\bowtie) \cdot$ Ruven Stahns

Institut Primarstufe, Pädagogische Hochschule der FHNW, Hofackerstr. 30, 4132 Muttenz, Schweiz

E-Mail: maja.wipraechtiger@fhnw.ch

Susanne Riegler

Erziehungswissenschaftliche Fakultät, Grundschuldidaktik Deutsch, Universität Leipzig,

Marschnerstraße 31, 04109 Leipzig, Deutschland 


\title{
Subject-specific instructional quality in the german language und literature education
}

\begin{abstract}
Based on the finding that German language and literature education only implicitly focused on teaching quality in the past, this article presents trends in German language and literature education research with regard to teaching quality. It can be stated that there are now several studies that are connected to the findings of general research on teaching quality and specify them for German lessons, especially with regard to the dimension "cognitive activation". These are, on the one hand, survey studies in the context of large-scale assessments, which relate rather general features of teaching quality to German lessons and are so called subject-related. On the other hand, there are video studies in which cognitive activation is no longer conceptualized and operationalized in a general way, but in a subject-specific way. In the second part of the article, the concept of subject-specific instructional quality used in the "Profess-R" project is shortly presented. Based on the belief that the specification of cognitive activation is not sufficient for an adequate subject-specific definition of teaching quality, a model is presented in which generic, subject-related and subject-specific aspects are interwoven on several levels.
\end{abstract}

Keywords Subject-specific definition of teaching quality · German language and literature education $\cdot$ Cognitive activation $\cdot$ Spelling lessons

\section{Zum Stand der Diskussion in der Deutschdidaktik}

Die Fachdidaktik Deutsch beschäftigt sich traditionell intensiv mit der Frage, was „guter Deutschunterricht“ ist. Im Bemühen um einen gegenstands- und lernerangemessenen Unterricht entwickelt sie Konzepte und Methoden für das Lehren und Lernen in den verschiedenen Lernbereichen des Deutschunterrichts. Die vorgeschlagenen Konzepte und Methoden werden vornehmlich theoretisch begründet und häufig kontrovers diskutiert, da kaum empirische Befunde zur ihrer Wirksamkeit vorliegen (vgl. exemplarisch für den Lernbereich „Richtig schreiben“ Kruse und Reichardt 2016). Gleichwohl existieren in der Zwischenzeit Interventions- und Methodenvergleichsstudien, in denen Unterrichts- und Förderkonzepte für die verschiedenen Lernbereiche des Deutschunterrichts evaluiert werden (vgl. z. B. zum ,strukturorientierten Rechtschreiblernen“ Bangel und Müller 2018; zum Vergleich unterschiedlicher Konzepte für den Schriftspracherwerb Weinhold 2009). In diesen Studien werden Fragen der Unterrichtsqualität meist nur implizit verhandelt, ohne dass auf das Konstrukt „Unterrichtsqualität“ aus der empirischen Bildungsforschung zurückgegriffen wird. Die Qualität des Unterrichts wird hier im Wesentlichen an der Verwendung bestimmter Ansätze oder Methoden festgemacht, die sich - bei konzepttreuer Umsetzung - für den Kompetenzerwerb der Lernenden als effektiv erwiesen haben. Dies muss aber zu kurz greifen, da Merkmale der Prozessqualität des Unterrichts dabei nicht berücksichtigt werden.

Vor diesem Hintergrund ist es nur folgerichtig, dass die Deutschdidaktik in den letzten Jahren zunehmend Anschluss an die allgemeine (bislang stark mathematisch- 
naturwissenschaftlich geprägte) empirische Unterrichtsforschung sucht und deren Befunde auf den Deutschunterricht bezieht. In einer Reihe von Publikationen werden Merkmale, die sich in anderen Fächern als lernwirksam erwiesen haben, in ihrer Bedeutung für den Deutschunterricht reflektiert, ohne jedoch mit empirischen Daten zu prüfen, ob diese Merkmale im Deutschunterricht zu beobachten sind und/oder mit Lernerfolgen zusammenhängen (Bremerich-Vos 2000; Eikenbusch 2005; Grundler 2018).

Vor allem aber gibt es inzwischen einige empirische Studien zur Qualität des Deutschunterrichts, die dezidiert an Befunde der allgemeinen Unterrichtsqualitätsforschung anschließen und sich dabei meist am Konzept der drei Basisdimensionen orientieren: Unterrichts- bzw. Klassenführung, konstruktive Unterstützung bzw. unterstützendes Lernklima sowie kognitive Aktivierung (Klieme et al. 2006; Lipowsky und Bleck 2019; Praetorius et al. 2020). Im Fokus steht dabei die ,,kognitive Aktivierung", die für die fachlichen Lernerfolge von Schüler(inne)n besonders bedeutsam sein dürfte (Hanisch 2018; Stahns et al. 2020). Da bei der kognitiven Aktivierung „die Beziehung zwischen Lernenden und Lerngegenstand“ (Winkler 2017, S. 79) im Mittelpunkt steht, wird für diese Dimension die Notwendigkeit einer für den Deutschunterricht spezifischen Operationalisierung betont.

Bei den derzeit vorliegenden empirischen Arbeiten handelt es sich zunächst um Fragebogenstudien aus dem Kontext von Large-Scale-Assessments. Dabei wurde z. B. in IGLU 2006 (Lankes und Carstensen 2007) zunächst versucht, das kognitive Aktivierungspotenzial des Unterrichts mithilfe von Selbstauskünften der Lehrpersonen (etwa über die Häufigkeit des Einsatzes von Lesestrategien) zu erfassen. Da damit aber keine Aussagen über die Qualität der konkreten Umsetzung möglich sind, wurden in späteren Erhebungsrunden Items zur Prozessqualität aus anderen Fächern für die Lehrer- und Schülerfragebögen übernommen und auf das Fach Deutsch bezogen (Stahns und Rieser 2018; Stahns et al. 2017, 2020). Diese Items sind nicht im engeren Sinne fachspezifisch, weil sie für jedes Fach gelten können, sie sind aber fachbezogen, d.h. im Hinblick auf ihre Relevanz für das Fach Deutsch ausgewählt und in der Itemformulierung auf den Deutschunterricht ausgerichtet (z.B. „Unser Deutschlehrer/unsere Deutschlehrerin möchte, dass wir unsere Antworten erklären.").

Darüber hinaus liegen (Video-)Studien zu verschiedenen Lernbereichen des Deutschunterrichts vor, die sich um eine Erfassung des Potenzials zur kognitiven Aktivierung bemühen (Stahns 2013; Lotz 2016; Winkler 2017; Hanisch 2018; ähnlich „Kompetenzorientierung“ bei Kleinbub 2010). Fokussiert werden dabei meist Merkmale des Unterrichtsgesprächs und/oder der eingesetzten Aufgaben, die letztendlich fächerübergreifende Gültigkeit haben und hier fachbezogen, also unter „Berücksichtigung fachspezifischer Relevanzsetzungen“ (Winkler 2017, S. 93) ausgewählt und eingesetzt werden (Pracht und Löffler 2012; Stahns 2013; Winkler 2017). Lotz (2016) macht dagegen kognitive Aktivierung im Leseunterricht u.a. an der Vermittlung von Lesestrategien fest und versucht damit, das Potenzial zur kognitiven Aktivierung fachspezifisch zu operationalisieren. Anders als im Rahmen von Fragebogenerhebungen bei IGLU wird auf der Basis von Videoaufzeichnungen z. B. erfasst, ob Lehrpersonen Lesestrategien explizit erläutern oder modellieren. 
Jüngst wird in der Deutschdidaktik thematisiert, dass mit der alleinigen Fokussierung auf die Dimension „Kognitive Aktivierung“ zahlreiche Merkmale fachlicher Unterrichtsqualität unberücksichtigt bleiben (Winkler 2020, S. 25; Stahns im Druck). Hier folgt die Deutschdidaktik der Diskussion um die Weiterentwicklung bzw. Ergänzung des dreidimensionalen Modells von Unterrichtsqualität um weitere Dimensionen fachbezogener oder fachspezifischer Unterrichtsqualität (Lipowsky und Bleck 2019), die zumindest für einige Fächer (Mathematik, Sport u.a.m.) im Sinne eines Frameworks konkretisiert werden konnten (Praetorius et al. 2020). Eine systematische Aufarbeitung der für das Fach Deutsch notwendigen fachspezifischen Ergänzungen eines solchen Frameworks steht aus. Es gibt bislang nur einige Fallstudien zum Deutschunterricht, in denen fachspezifische Aspekte von Unterrichtsqualität berücksichtigt werden (z. B. Bremerich-Vos 2006; Stahns und BremerichVos 2013; Stahns im Druck). Der Diskurs darüber, in welcher Art und auf welcher Ebene die postulierte Fachspezifik für den Deutschunterricht zu konzeptualisieren wäre, steht noch am Anfang. Ungeklärt ist auch die Frage, ob sich solche fachspezifischen Aspekte überhaupt auf den Deutschunterricht als Gesamten beziehen lassen oder ob sie nicht vielmehr für die einzelnen Lernbereiche bestimmt werden müssen.

\section{Eigene Positionierung: Fachspezifische Unterrichtsqualität im Projekt ,Profess-R“}

Ein Versuch, ein entsprechend ergänztes Modell von Unterrichtsqualität zu entwickeln, wurde in der ländervergleichenden Videostudie „Professionelle Kompetenzen und Unterrichtshandeln von Primarlehrpersonen im Lernbereich Rechtschreiben“" (Profess-R) unternommen. Leitend für diese Entwicklung waren fachliche Befunde zum Orthografieerwerb und zum Rechtschreibunterricht, normative Überlegungen zu ,gutem“ Rechtschreibunterricht sowie Befunde zur Unterrichtsqualität im Deutschunterricht und in anderen Fächern. Dabei zeigte sich, dass mit den drei Basisdimensionen relevante fachliche Aspekte für die Einschätzung der Unterrichtsqualität im Deutschunterricht nicht hinreichend erfasst werden können. In Anlehnung an Praetorius und Charalambous (2018) wurde daher die Dimension „Auswahl und Präsentation des Lerngegenstandes“ ergänzt und für den Rechtschreibunterricht (bzw. den Lerngegenstand Doppelkonsonantenschreibung) konkretisiert. Aus fachlicher Sicht ist es höchst relevant, welche Aspekte des Lerngegenstands ausgewählt werden und wie dieser im Unterricht präsentiert wird. Damit werden fachliche Lerngelegenheiten geschaffen, die eine unerlässliche Voraussetzung für einen gesteuerten Kompetenzerwerb im Lernbereich „Richtig schreiben“ darstellen. Entscheidend ist dabei die fachliche Qualität ihrer Ausgestaltung, die mit dieser Dimension eingeschätzt werden soll.

Da wir zudem zwischen sozio-emotionaler und kognitiver Unterstützung differenzieren (vgl. Kleickmann et al. 2020), setzen wir ein Modell von Unterrichtsqualität mit den folgenden fünf Dimensionen an: 1. Effiziente Klassenführung, 2. Auswahl und Präsentation des Lerngegenstands, 3. Sozio-emotionale Unterstützung, 4. Kognitive Aktivierung und 5. Kognitive Unterstützung. 
In diesem Modell greifen generische, fachbezogene und fachspezifische Aspekte auf mehreren Ebenen ineinander. Auf welcher Ebene Fachlichkeit eine Rolle spielt, ist für jede (Sub-)Dimension im Einzelfall zu klären. Dies soll kurz an der Operationalisierung der Dimension „Auswahl und Präsentation des Lerngegenstands“ erläutert werden. Es handelt sich grundsätzlich um eine hybride Dimension, die neben den bereits erwähnten fachlichen Aspekten auch generische Merkmale umfasst. So sind die Subdimensionen zwar generisch formuliert, werden hier aber fachbezogen eingesetzt, da mit dem Lerngegenstand bzw. der fachlichen Lerngelegenheit das Fach und seine Inhalte ins Spiel kommen: 1. Inhaltlicher Fokus der fachlichen Lerngelegenheiten, 2. Situierung des Lerngegenstands, 3. Klare und strukturierte Präsentation des Lerngegenstands und 4. Fachlich korrekte Präsentation des Lerngegenstands.

Die Fachspezifik zeigt sich dabei teilweise auf der Ebene der Items, teilweise aber auch erst auf der Ebene der Auswahl und Beschreibung der Indikatoren. Items wie „Der Lehrperson unterlaufen im Unterricht keine fachlichen Fehler" sind nur fachbezogen, bei den Indikatoren kommen jedoch auch fachspezifische Gesichtspunkte, etwa die Definition, was als fachlicher Fehler zählt, zum Tragen. Wird die Passung des im Unterricht verwendeten Wortmaterials analysiert (Item: „Das Wortmaterial repräsentiert den Lerngegenstand in angemessener Weise."), ist dies genuin fach- bzw. sogar gegenstandsspezifisch.

Eine angemessene Berücksichtigung fachlicher Aspekte von Unterrichtsqualität ist aus unserer Sicht nur durch die hier angedeutete Integration generischer, fachbezogener und fachspezifischer Aspekte möglich. Neben den Dimensionen „Kognitive Aktivierung“ und „Kognitive Unterstützung“ braucht es dazu eine Dimension wie „Auswahl und Präsentation des Lerngegenstands“, mit deren Hilfe die Gegenstandsangemessenheit des Unterrichts erfasst werden kann. Inwiefern mit einer solchen fachlichen Ausgestaltung von Dimensionen, Items oder Indikatoren die damit erfassten Konstrukte in den unterschiedlichen Lernbereichen des Deutschunterrichts - und erst recht in anderen Fächern - noch vergleichbar sind, muss aktuell offenbleiben. Es ist zu wünschen, dass diese Fragen auch in der Deutschdidaktik diskutiert und für die unterschiedlichen Lernbereiche und deren Vergleich untereinander aufgearbeitet werden. Zudem gilt es empirisch zu prüfen, ob sich die so dimensionierte fachspezifische Prozessqualität als bedeutsam für das Lernen im Deutschunterricht erweist. Die Deutschdidaktik täte gut daran, in diese Diskussion verstärkt einzusteigen und ihre ureigenen Kompetenzen, wie das Wissen um die Gegenstände und deren Erwerbsprozesse, differenziert und selbstbewusst in die Erforschung der Unterrichtsqualität einzubringen.

Funding Open access funding provided by FHNW University of Applied Sciences and Arts Northwestern Switzerland

Open Access Dieser Artikel wird unter der Creative Commons Namensnennung 4.0 International Lizenz veröffentlicht, welche die Nutzung, Vervielfältigung, Bearbeitung, Verbreitung und Wiedergabe in jeglichem Medium und Format erlaubt, sofern Sie den/die ursprünglichen Autor(en) und die Quelle ordnungsgemäß nennen, einen Link zur Creative Commons Lizenz beifügen und angeben, ob Änderungen vorgenommen wurden. 
Die in diesem Artikel enthaltenen Bilder und sonstiges Drittmaterial unterliegen ebenfalls der genannten Creative Commons Lizenz, sofern sich aus der Abbildungslegende nichts anderes ergibt. Sofern das betreffende Material nicht unter der genannten Creative Commons Lizenz steht und die betreffende Handlung nicht nach gesetzlichen Vorschriften erlaubt ist, ist für die oben aufgeführten Weiterverwendungen des Materials die Einwilligung des jeweiligen Rechteinhabers einzuholen.

Weitere Details zur Lizenz entnehmen Sie bitte der Lizenzinformation auf http://creativecommons.org/ licenses/by/4.0/deed.de.

\section{Literatur}

Bangel, M., \& Müller, A. (2018). Strukturorientiertes Rechtschreiblernen. Ergebnisse einer Interventionsstudie zur Wortschreibung in Klasse 5 mit Blick auf schwache Lerner/-innen. Didaktik Deutsch, 45, 29-49.

Bremerich-Vos, A. (2000). Was ist guter Deutschunterricht? In Landesinstitut für Schule und Weiterbildung (Hrsg.), Was ist guter Fachunterricht? Beiträge zur fachwissenschaftlichen Diskussion (S. 54-74). Bönen: Kettler.

Bremerich-Vos, A. (2006). Zur Videographie von (Deutsch-)Unterricht. Anmerkungen zur Vermittelbarkeit von linguistischer, sprachdidaktischer und pädagogisch-psychologischer Unterrichtsforschung. In I. Hosenfeld \& F.-W. Schrader (Hrsg.), Schulische Leistung. Grundlagen, Bedingungen, Perspektiven (S. 243-262). Münster: Waxmann.

Eikenbusch, G. (2005). Qualität im Deutschunterricht der Sekundarstufe I und II. Berlin: Cornelsen Scriptor.

Grundler, E. (Hrsg.). (2018). Wirksamer Deutschunterricht. Baltmannsweiler: Schneider Verlag Hohengehren.

Hanisch, A.-K. (2018). Kognitive Aktivierung im Rechtschreibunterricht. Eine Interventionsstudie in der Grundschule. Münster: Waxmann.

Kleickmann, T., Steffensky, M., \& Praetorius, A.-K. (2020). Quality of teaching in science education: More than three basic dimensions? Zeitschrift für Pädagogik, Beiheft, 66, 37-55.

Kleinbub, I. D. (2010). Unterrichtsqualität im Leseunterricht. Eine videobasierte Analyse in vierten Klassen. Trier: Wissenschaftlicher Verlag.

Klieme, E., Lipowsky, F., Rakoczy, K., \& Ratzka, N. (2006). Qualitätsdimensionen und Wirksamkeit von Mathematikunterricht. Theoretische Grundlagen und ausgewählte Ergebnisse des Projekts „Pythagoras“. In M. Prenzel \& L. Allolio-Näcke (Hrsg.), Untersuchungen zur Bildungsqualität von Schule. Abschlussbericht des DFG-Schwerpunktprogramms BiQua (S. 127-146). Münster: Waxmann.

Kruse, N., \& Reichardt, A. (Hrsg.). (2016). Wie viel Rechtschreibung brauchen Grundschulkinder? Positionen und Perspektiven zum Rechtschreibunterricht in der Grundschule. Berlin: Erich Schmidt.

Lankes, E.-M., \& Carstensen, C.H. (2007). Der Leseunterricht aus der Sicht der Lehrkräfte. In W. Bos, S. Hornberg, K.-H. Arnold, G. Faust, L. Fried, E.-M. Lankes, K. Schwippert \& R. Valtin (Hrsg.), IGLU 2006. Lesekompetenzen von Grundschulkindern in Deutschland im internationalen Vergleich (S. 161-193). Münster: Waxmann.

Lipowsky, F., \& Bleck, V. (2019). Was wissen wir über guten Unterricht? - Ein Update. In U. Steffens \& R. Messner (Hrsg.), Konzepte und Bilanzen gelingenden Lehrens und Lernens - Grundlagen der Qualität von Schule (Bd. 3, S. 219-249). Münster: Waxmann.

Lotz, M. (2016). Kognitive Aktivierung im Leseunterricht in der Grundschule. Eine Videostudie zur Gestaltung und Qualität von Leseübungen im ersten Schuljahr. Wiesbaden: Springer.

Pracht, H., \& Löffler, C. (2012). Analyse des kognitiven Aktivierungspotenzials von Aufgaben zum Orthographieerwerb. In A. Ballis \& A. Peyer (Hrsg.), Lernmedien und Lernaufgaben im Deutschunterricht (S. 49-67). Bad Heilbrunn: Klinkhardt.

Praetorius, A.-K., \& Charalambous, C. Y. (2018). Classroom observation frameworks for studying instructional quality: looking back and looking forward. ZDM Mathematics Education, 50(3), 535-553.

Praetorius, A.-K., Herrmann, C., Gerlach, E., Zülsdorf-Kersting, M., Heinitz, B., \& Nehring, A. (2020). Unterrichtsqualität in den Fachdidaktiken im deutschsprachigen Raum - zwischen Generik und Fachspezifik. Unterrichtswissenschaft, 48, 409-466.

Stahns, R. (2013). Kognitive Aktivierung im Grammatikunterricht. Videoanalysen zum Deutschunterricht. Baltmannsweiler: Schneider Verlag Hohengehren. 
Stahns, R., \& Bremerich-Vos, A. (2013). Aspekte empirischer Unterrichtsforschung. Zur Videographie bzw. Transkription von Grammatikunterricht. In K.-M. Köpcke \& A. Ziegler (Hrsg.), Schulgrammatik und Sprachunterricht im Wandel (S. 151-175). Berlin, Boston: De Gruyter.

Stahns, R., \& Rieser, S. (2018). Qualität des Leseunterrichts in vierten Klassen in der Grundschule unter den Bedingungen von Mehrsprachigkeit. Ergebnisse der Lehrkräftebefragung von IGLU 2011. Zeitschrift für Grundschulforschung, 11(1), 131-145.

Stahns, R., Rieser, S., \& Hußmann, A. (2020). Können Viertklässlerinnen und Viertklässler Unterrichtsqualität valide einschätzen? Ergebnisse zum Fach Deutsch. Unterrichtswissenschaft. https://doi.org/ 10.1007/s42010-020-00084-6.

Stahns, R., Rieser, S., \& Lankes, E.-M. (2017). Unterrichtsführung, Sozialklima und kognitive Aktivierung im Deutschunterricht in vierten Klassen. In A. Hußmann, H. Wendt, W. Bos, A. Bremerich-Vos, D. Kasper, E.-M. Lankes, N. McElvany, T.C. Stubbe \& R. Valtin (Hrsg.), IGLU 2016. Lesekompetenzen von Grundschulkindern in Deutschland im internationalen Vergleich (S. 251-277). Münster: Waxmann.

Stahns, R. Zur empirischen Erforschung der Prozessqualität des Grammatikunterrichts. In H. Gornik \& I. Rautenberg (Hrsg.), Sprachreflexion und Grammatikunterricht 3., überarb. und erweit. Aufl. Baltmannsweiler: Schneider Verlag Hohengehren.

Weinhold, S. (2009). Effekte fachdidaktischer Ansätze auf den Schriftspracherwerb in der Grundschule. Lese- und Rechtschreibleistungen in den Jahrgangsstufen 1-4. Didaktik Deutsch, 27, 53-75.

Winkler, I. (2017). Potenzial zu kognitiver Aktivierung im Literaturunterricht. Fachspezifische Profilierung eines prominenten Konstrukts der Unterrichtsforschung. Didaktik Deutsch, 43, 78-97.

Winkler, I. (2020). Cognitive activation in L1 literature classes. A content-specific framework for the description of teaching quality. L1-Educational Studies in Language and Literature, 20, 1-32. https:// doi.org/10.17239/L1ESLL-2020.20.01.03. 